\title{
Robotic surgery in obese patients with early-stage endometrial cancer
}

\author{
Yanyan Zhao ${ }^{1,2}$, Zhongyu Liu ${ }^{1}$, Ling Yu ${ }^{1}$, Sai Liü ${ }^{3}$, Hong Yan ${ }^{1}$, Yan Zhang ${ }^{2}$, Yuanqing Yao ${ }^{1}$ \\ ${ }^{1}$ Department of Gynaecology and Obstetrics, The First Medical Center of PLA General Hospital, Beijing, China \\ 2Department of Gynaecology and Obstetrics, The First Hospital of Jiamusi University, Jiamusi, China \\ ${ }^{3}$ Department of Gynaecology and Obstetrics, Gan Mei Hospital of the First People's Hospital, Kunming, China
}

Videosurgery Miniinv 2020; 15 (1): 171-175

DOI: https://doi.org/10.5114/wiitm.2019.89337

\begin{abstract}
Aim: The objective of this study was to assess the clinical effectiveness of robotic surgery for obese patients (body mass index $(B M I) \geq 30 \mathrm{~kg} / \mathrm{m}^{2}$ ) with early stage endometrial cancer.

Material and methods: This study is a retrospective review of women who underwent robotic surgery for early-stage endometrial cancer from 2008 to 2017. Patients were subdivided into those with BMI $<30 \mathrm{~kg} / \mathrm{m}^{2}$ (group 1), and those with $B M I \geq 30 \mathrm{~kg} / \mathrm{m}^{2}$ (group 2). Basic demographics and perioperative period outcomes were extracted from the medical records and compared.

Results: Group 1 included fifty patients and group 2 included 24 patients. There were no significant differences in surgical outcomes or complication rates between the two groups ( $p>0.05$ for all). There were no differences in pelvic nodal counts or length of stay.

Conclusions: Robotic surgery was found to be feasible and safe for obese patients with endometrial cancer. Its widespread application needs a larger sample with longer follow-up.
\end{abstract}

Key words: robotic surgery, obese, endometrial cancer, body mass index.

\section{Introduction}

Endometrial cancer is one of the most common gynecological malignancies in the world [1]. Women have a lifetime risk of 1 in 38 of developing uterine cancer [2]. Obesity is a recognized risk factor for multiple cancers, cancer-related deaths and all causes of death. Previous studies have found that obesity and increasing body mass index (BMI) are most closely related to the incidence and mortality of endometrial cancer [3-5]. Obesity increases the risk of cancer tenfold, and in $40 \%$ of patients with endometrial cancer it is due to obesity [6].

Obese patients face unique surgical and technical challenges related to their multiple perioperative complications and difficult surgical treatment [7]. As the patient becomes fatter, to achieve the same therapeutic effect, several surgical techniques need to be evaluated. Fortunately, laparoscopic surgery reduces some of the surgical difficulties inherent in dealing with obese patients [8]. Some studies have demonstrated the benefits of robotic surgery and robotic techniques for endometrial cancer compared with laparotomy [9-11]. Studies have also demonstrated that robotic surgery for obese patients is associated with higher costs due to greater equipment use and longer operating times [12-14]. However, no reports have studied the use of robotic surgery for obese patients with endometrial cancer to compare robotic surgery for normal BMI endometrial cancer patients.

\section{Address for correspondence}

Prof. Yuanqing Yao, Department of Gynaecology and Obstetrics, The First Medical Center of PLA General Hospital, Beijing, China,

e-mail: yqyao301hp@163.com 
The primary objective of this study was to compare perioperative outcomes of robotic surgery for obese patients with endometrial cancer and normal $\mathrm{BMI}$ patients. This is the first study of robotic surgery for obese patients with endometrial cancer in China.

\section{Aim}

The aim of this study was to assess the clinical curative effective of robotic surgery for obese patients $\left(\mathrm{BMI} \geq 30 \mathrm{~kg} / \mathrm{m}^{2}\right)$ with early stage endometrial cancer.

\section{Material and methods}

This study obtained the institutional review board approval of our hospital, We prospectively collected 74 patients with early stage endometrial cancer who accepted robotic surgery. Robot cases were collected when robotic surgery was introduced into this practice from January 2008 to December 2017. Robot cases are completed by a team of gynecologic oncologists who are qualified to use the availability of robotic platforms and robotic platforms themselves in our hospital. There are no other criteria for selecting surgical methods. During informed consent, patients were counseled that the RSS platform would be a novel therapeutic strategy for early stage endometrial cancer. Surgical consent was obtained from all patients.

Eligible patients have no tumors other than endometrial cancer and no cardiovascular, pulmonary or endocrine diseases. Eligibility criteria included pelvic magnetic resonance imaging (MRI) and ultrasonography in patients with endometrial carcinoma suspected of early lesions, as well as patients with preoperative biopsies showing endometrioid histology. All patients provided detailed consent to undergo robotic surgery. The included patients were divided into two groups based on their BMI. Patients with BMI less than $30 \mathrm{~kg} / \mathrm{m}^{2}$ were included in group 1 , and those with BMI equal to or greater than $30 \mathrm{~kg} / \mathrm{m}^{2}$ were included in group 2.

Demographic data, operative data, perioperative complications, and the pathologic result were collected and analyzed. Demographic data included age, BMI, the patient's comorbidities, the history of surgery, and menopausal status. Surgical statistics include the operation time, conversion rate, and estimated blood loss. Surgical complications include blood loss requiring blood transfusion and major nerve, vascular, gastrointestinal or urinary tract injuries. Postoperative complications include unplanned readmission within 30 days after the operation, wound complications (seroma, hematoma, wound separation, wound infection), venous thromboembolism, or any other major event that can be considered a direct result of the operation.

All patients accepted antibiotic prophylaxis before surgery, and bowel preparation with polyethylene glycol electrolyte powder. All patients accepted three times cleansing of the vagina with povidone iodine solution preoperatively. Stretch socks were used to prevent venous thrombosis and Foley catheters were placed intraoperatively. All patients underwent robotic hysterectomy bilateral adnexectomy and pelvic lymphadenectomy using the Da Vinci Si surgical system (Intuitive Surgical Inc., Sunnyvale, CA). All operations were performed under anesthesia with endotracheal intubation. It was placed in the position of the sarcophagus and its arms were sandwiched on both sides. Three robotic arms were used and five trocars in all. The location and layout of trocars on the abdominal wall were consistent with the results of our previous research on robotic cervical cancer [15].

\section{Statistical analysis}

SPSS version 16.0 (SPSS Inc, Chicago, IL, USA) was used for statistical analyses. The continuous variables with a normal distribution are represented as the mean (range), and the categorical variables are represented as the absolute quantity (percentage). We used Student's $t$ test to compare continuous variables with a normal distribution, and the $\chi^{2}$ test or Fisher's exact test was used to compare categorical variables. $P<0.05$ was considered statistically significant.

\section{Results}

There were 74 patients included this study between January 2008 and December 2017 in our hospital. All patients met the inclusion criteria. They all accepted robotic surgery for endometrial cancer. Base on the BMI of patients, all the included cases were divided into two groups. Fifty $(67.6 \%)$ patients were included in group 1 with $\mathrm{BMI}<30 \mathrm{~kg} / \mathrm{m}^{2}$, and $24(33.4 \%)$ patients with $\mathrm{BMI} \geq 30 \mathrm{~kg} / \mathrm{m}^{2}$ were included in group 2. Figure 1 shows the flow chart of inpatients. 


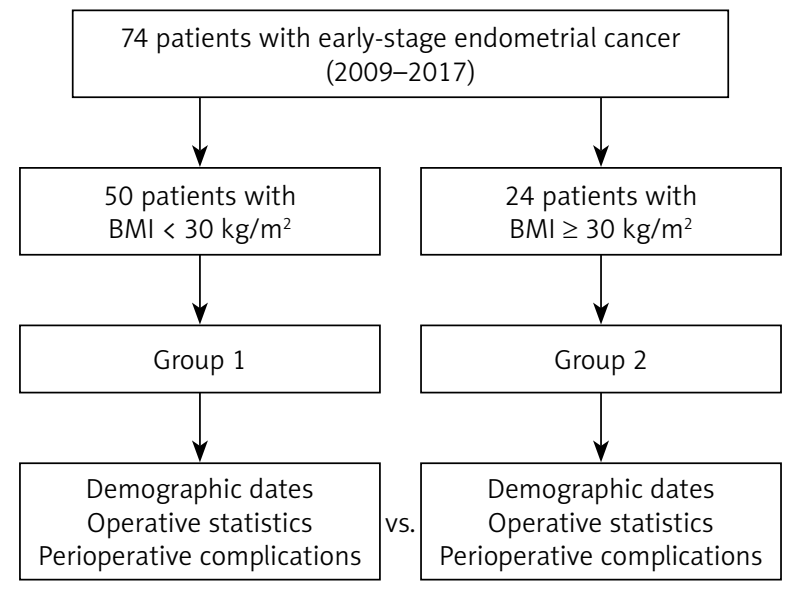

Figure 1. Flow chart of inpatients

The characteristics of the selected patients are shown in Table I. The median age of group 1 was 61 years (36 to 77 years). The median age of group 2 was 62 years (range: $30-76$ years). Comparing the ages of the two groups of patients, there was no significant difference. The two groups had similar previous pelvic surgery history. BMI was higher in group 2 than in group 1 (35.0 vs. $26 \mathrm{~kg} / \mathrm{m}^{2}$, respectively; $p<0.05)$. There was no case that had contraindications to robotic surgery in either group.

The operative and pathologic outcomes of included women are summarized in Table II. There was no difference between the groups in the International Federation of Obstetrics and Gynecology (FIGO) stage. The number of total nodes that were retrieved was 16 (11-23) in women of group 1 vs. 14 (13-25) nodes in group 2; there was no significant difference in the number of pelvic nodes. The median operative time of group 1 was 190 min (range: 150-240 min) and the median estimated blood loss was $75 \mathrm{ml}$ (range: 30-150 ml). The length of hospital stay of group 1 was 11 days (range: 9-13 days). The median operative time of group 2 was 180 min (range: 120$270 \mathrm{~min}$ ) and the median estimated blood loss was $90 \mathrm{ml}$ (range: 30-200 ml). The duration of hospitalization of group 2 was 11 days (8-15 days). There was no difference in terms of operation time, estimated blood loss or length of hospital stay between the two groups.

No ureteral injury, bladder injury, bowel injury, hernia or dehiscence, infection, or conversion to laparotomy was noted intraoperatively (Table III). There was no significant difference in the incidence of intraoperative complications between the two
Table I. Characteristics of included patients

\begin{tabular}{|lcc|}
\hline Variables & $\begin{array}{c}\text { Group 1 } \\
(n=50)\end{array}$ & $\begin{array}{c}\text { Group 2 } \\
(n=24)\end{array}$ \\
\hline BMI $\left[\mathrm{kg} / \mathrm{m}^{2}\right]$ & $26(23-29)$ & $35(30-42)^{*}$ \\
\hline Age, median (range) [years] & $61(36-77)$ & $62(30-76)$ \\
\hline Post-menopausal, $n(\%)$ & $20(40)$ & $12(50)$ \\
\hline Prior abdominal surgery, $n(\%)$ & $10(20)$ & $5(20.8)$ \\
\hline $\begin{array}{l}\text { Contraindication for robotic } \\
\text { surgery, } n(\%)\end{array}$ & $0(0)$ & $0(0)$ \\
\hline
\end{tabular}

$B M I$ - body mass index. Data are expressed as number (\%) or median (range); * $p<0.05$.

Table II. Operative and pathologic outcomes among women who underwent surgery

\begin{tabular}{|lcc|}
\hline Variables & $\begin{array}{c}\text { Group } \\
(n=50)\end{array}$ & $\begin{array}{c}\text { Group 2 } \\
(n=24)\end{array}$ \\
\hline FIGO stage: & 8 & 3 \\
\hline IA & 19 & 10 \\
\hline IB & 22 & 10 \\
\hline IIA & 1 & 1 \\
\hline IIB & 0 & 0 \\
\hline Total pelvic nodes & $16(11-23)$ & $14(13-25)$ \\
\hline Operative time [min] & $190(150-240)$ & $180(120-270)$ \\
\hline Estimated blood loss [ml] & $75(30-150)$ & $90(30-200)$ \\
\hline Length of hospital stay [days] & $11(9-13)$ & $11(8-15)$ \\
\hline
\end{tabular}

Data are expressed as number (\%) or median (range). ${ }^{*} P<0.05$. FIGO - International Federation of Gynecology and Obstetrics.

Table III. Intraoperative and postoperative complications

\begin{tabular}{|lcc|}
\hline Variables & $\begin{array}{c}\text { Group } 1 \\
(n=50)\end{array}$ & $\begin{array}{c}\text { Group 2 } \\
(n=24)\end{array}$ \\
\hline Ureteral injury $(n / N)$ & $0(0 \%)$ & $0(0 \%)$ \\
\hline Bladder injury $(n / N)$ & $0(0 \%)$ & $0(0 \%)$ \\
\hline Bowel injury $(n / N)$ & $0(0 \%)$ & $0(0 \%)$ \\
\hline Blood transfusion $(n / N)$ & $0(0 \%)$ & $0(0 \%)$ \\
\hline Hernia/dehiscence $(n / N)$ & $0(0 \%)$ & $0(0 \%)$ \\
\hline Infection $(n / N)$ & $0(0 \%)$ & $0(0 \%)$ \\
\hline Lymphedema $(n / N)$ & $3(6 \%)$ & $1(4.2 \%)$ \\
\hline Voiding dysfunction $(n / N)$ & $0(0 \%)$ & $0(0 \%)$ \\
\hline Venous thromboembolism $(n / N)$ & $0(0 \%)$ & $0(0 \%)$ \\
\hline Wound complications $(n / N)$ & $0(0 \%)$ & $0(0 \%)$ \\
\hline Conversion $(n / N)$ & $0(0 \%)$ & $0(0 \%)$ \\
\hline
\end{tabular}

Data are expressed as number (\%). ${ }^{*} P<0.05$. 
groups ( $p>0.05)$. There were no postoperative complications such as intestinal trauma or obstruction, incisional hernia or dehiscence and reoperation occurred in either group. There were 3 patients in group 1 and one in group 2 who had a symptomatic lymphocyst; there was no difference between the two groups. No patient had venous thrombosis in the perioperative period.

\section{Discussion}

Surgical treatment has always been the cornerstone of early treatment of endometrial cancer. A variety of surgical methods are available for gynecologic oncologists through the newly developed minimally invasive surgical approach. In addition, with the increasing number of obese patients, the choice of surgical methods is becoming more and more important.

This study data suggest that robotic hysterectomy bilateral adnexectomy and pelvic lymphadenectomy for obese patients with early-stage endometrial cancer are technically feasible and safe. The operative time and estimated blood loss of obese patients were similar to patients with $\mathrm{BMI}<30 \mathrm{~kg} / \mathrm{m}^{2}$ with early-stage endometrial cancer. It has been suggested that the number of resected lymph nodes is the most important parameter for lymphadenectomy. The pelvic lymph node count and the length of hospital stay of patients in group 2 were similar to those observed in group 1. Especially, the incidence of surgical complications was low in both groups. Previous studies have found that obese patients who underwent pelvic surgery have longer operations, more intraoperative bleeding, and a higher incidence of complications, and the pelvic surgery for obese patients is technically challenging [16]. This is inconsistent with our findings. We obtained better results, which might be due to the advantages of $\mathrm{Da}$ Vinci robotics, and the surgical team's proficiency in gynecological operations using the Da Vinci robot.

In 2005, the American Food and Drug Administration (FDA) approved the robotic surgery system for gynecological treatment, which has had far-reaching significance for the surgical treatment of gynecologic malignancies. Compared with conventional surgical approaches, robotic surgery has various differences. First, the robotic surgery system has three-dimensional stereo vision. Second, the operation of the robotic arm is superior in dexterity and precision, due to the ability of the arm to rotate 360 degrees. Based on the above advantages, the robotic surgery system has become widely accepted by gynecologic oncologists for treatment of gynecologic malignancies. The popularity of robotics is reflected in the increase in its use, especially in the population of gynecological tumors. More than $66 \%$ of 386 gynecologists plan to increase the use of robotics in their practice, according to a Mabrouk et al. survey [17].

Boggess et al. compared open staging, laparoscopic staging and robotic staging of endometrial cancer; the advantages of robot technology were demonstrated. In their laparoscopic and robotic group comparison of 322 women, the $\mathrm{BMI}$ and pelvic lymph node count of the robot group were the highest, the blood loss of the robotic group was lowest, and the robotic group had the shortest hospitalization time. In addition, the incidence of postoperative complications in the robotic group was significantly reduced [18]. A study by Seamon et al. produced similar results. The BMI of the robotic cohort was significantly higher than that of the laparoscopic cohort (34 vs. 29, $p<0.001$ ), the robotic cohort had lower estimated blood loss and conversion to laparotomy rate, and robotic surgery had a shorter operating time [19]. These studies' results suggest that robots may be the first choice for the surgical approach of endometrial cancer. The question that followed was whether these developments were related to surgery in obese people.

In this study, the clinical outcome of robotic surgery for obese patients with early-stage endometrial cancer is consistent with the normal patients. There was no significant difference in operating time, estimated blood loss, inpatient time or perioperative complications between the two groups, and BMI is an exception. In this retrospective cohort study, robotics provided the same approach to the surgical staging of morbid obesity. There were no case transitions in this study, especially for our normal obese patients. There was no difference in dissected lymph node count between the two groups. Robotic surgery for obese endometrial cancer seems to be an attractive option. The shortcoming of this study is the absence of long-term follow-up results, and the long-term survival. It is an important index to evaluate the clinical effect of the surgery approach for malignant tumors. According to our data, robotic surgery was acceptable for surgical treatment of obese women with endometrial cancer. However, 
the limited number of included patients represents the main limitation of the study.

Widespread application of robotic surgery for obese patients with early stage endometrial cancer needs a larger sample with data from a longer follow-up. At the same time, the management of endometrial cancer should be personalized, taking into account the performance status of the patient, in particular in the case of elderly women $[20,21]$.

\section{Conclusions}

Robotic surgery for obese patients with early stage endometrial cancer is feasible and safe. Robotic surgery could be the better choice of treatment approach for obese patients with early stage endometrial cancer. Moreover, the robotic surgery system allows the surgeon to operate in a more comfortable situation. Widespread application of robotic surgery for obese patients with early stage endometrial cancer needs a larger sample with longer follow-up data.

\section{Acknowledgments}

Yanyan Zhao and Zhongyu Liu contribution equally for this article.

\section{Conflict of interest}

The authors declare no conflict of interest.

\section{References}

1. Sorosky J. Endometrial cancer. Obstet Gynecol 2012; 120: 383-97.

2. Siegel R, Naishadham J, Jemal A. Cancer statistics 2012. CA Cancer J Clin 2012; 62: 10-29.

3. Reeves GK, Pirie K, Beral V, et al. Cancer incidence and mortality in relation to body mass index in the Million Women Study: cohort study. BMJ 2007; 335: 1134.

4. Bessonova L, Marshall SF, Ziogas A, et al. The association of body mass index with mortality in the California Teachers Study. Int J Cancer 2011; 129: 2492-501.

5. Renehan AG, Tyson M, Egger M, et al. Body-mass index and incidence of cancer: a systematic review and meta-analysis of prospective observational studies. Lancet 2008; 371: 569-78.

6. Everett E, Tamimi H, Greer B, et al. The effect of body mass index on clinical/pathologic features, surgical morbidity, and outcome in patients with endometrial cancer. Gynecol Oncol 2003; 90: 150-7.

7. Chan JK, Cheung MK, Huh WK, et al. Therapeutic role of lymph node resection in endometrioid corpus cancer: a study of 12,333 patients. Cancer 2006; 107: 1823-30.

8. Obermair A, Manolitsas TP, Leung Y, et al. Total laparoscopic hysterectomy versus total abdominal hysterectomy for obese women with endometrial cancer. Int J Gynecol Cancer 2005; 15: 319-24.

9. Reynolds RK, Burke WM, Advincula AP. Preliminary experience with robot-assisted aparoscopic staging of gynecologic malignancies. JSLS 2005; 9: 149-58.

10. Peiretti M, Zanagnolo V, Bocciolone L, et al. Robotic surgery: changing the surgical approach for endometrial cancer in a referral cancer center. J Minim Invasive Gynecol 2009; 16: 427-31.

11. Lowe MP, Johnson PR, Kamelle SA, et al. A multiinstitutional experience with robotic assisted hysterectomy with staging for endometrial cancer. Obstet Gynecol 2009; 114: 236-43.

12. Hubens G, Balliu L, Ruppert M, et al. Roux-en-Y gastric bypass procedure performed with the da Vinci robot system: is it worth it? Surg Endosc 2008; 22: 1690-6.

13. Scozzari G, Rebecchi F, Millo P, et al. Robot-assisted gastrojejunal anastomosis does not improve the results of the laparoscopic Rouxen-Y gastric bypass. Surg Endosc 2011; 25: 597-603.

14. Barkun JS, Aronson JK, Feldman LS, et al. Evaluation and stages of surgical innovations. Lancet 2009; 374: 1089-96.

15. Zhongyu Liu, Xiuli Li ,Weiping Li, et al. Robotic nerve-sparing radical hysterectomy for locally advanced cervical cancer after neoadjuvant chemotherapy. Int J Gynaecol Obstet 2015; 131: 152-5.

16. Pavelka JC, Ben-Shachar I, Fowler JM, et al. Morbid obesity and endometrial cancer: surgical, clinical, and pathologic outcomes in surgically managed patients. Gynecol Oncol 2004; 95: 588-92.

17. Mabrouk M, Frumovitz M, Greer M, et al. Trends in laparoscopic and robotic surgery among gynecologic oncologists: a survey update. Gynecol Oncol 2009; 112: 501-5.

18. Boggess JF, Gehrig PA, Cantrell L, et al. A comparative study of 3 surgical methods for hysterectomy with staging for endometrial cancer: robotic assistance, laparoscopy, laparotomy. Am J Obstet Gynecol 2008; 199: 360.e1-9.

19. Seamon LG, Cohn DL, Henretta MS, et al. Minimally invasive comprehensive surgical staging for endometrial cancer: robotics or laparoscopy? Gynecol Oncol 2009; 113: 36-41.

20. Vitale SG, Capriglione S, Zito G, et al. Management of endometrial, ovarian and cervical cancer in the elderly: current approach to a challenging condition. Arch Gynecol Obstet 2019; 299: 299-315.

21. Bourgin C, Saidani M, Poupon C, et al. Endometrial cancer in elderly women: which disease, which surgical management? A systematic review of the literature. Eur I Surg Oncol 2016; 42: 166-75.

Received: 13.06 .2019 , accepted: 6.08.2019. 